\title{
Tchernobyl à l'Assistance publique Hôpitaux de Paris
}

\author{
A. LAUGIER ${ }^{\star \star}$, D. CAZEJUST ${ }^{\star \star \star}$ et B. GINDREY-VIE**
}

(Manuscrit reçu le 20 juillet 1987)

Dans l'année qui a suivi l'accident de Tchernobyl, des structures destinées à faire face à une situation identique en France se sont mises en place. Nous avons conduit, dans le cadre des travaux de la Commission des accidents collectifs, une enquête sur les moyens matériels et humains d'accueil à l'Assistance publique - Hôpitaux de Paris, des personnes irradiées et/ ou contaminées afin de répondre à la question suivante: quelle aurait été la capacité d'hospitalisation des hôpitaux de l'Assistance publique si un accident nucléaire était survenu le 26 avril 1987, soit un an jour pour jour après Tchernobyl?

Ce document n'envisage ni les moyens de décontamination sur place, ni ceux d'évacuation et d'acheminement des accidentés pour lesquels existe la procédure des SAMU (Services d'aide médicale urgente) ${ }^{(1)}$, celle du SCPRI (Service central de protection contre les rayonnements ionisants) ${ }^{(2)}$, le service des brûlés et de radiobiologie de l'hôpital Percy ${ }^{(3)}$, les capacités de l'Institut Gustave-Roussy ${ }^{(4)}$ et le Centre international de radiopathologie ${ }^{(5)}$.

* Communication présentée le 7 juillet 1987 à la Commission des accidents collectifs.

** Service de radiothérapie, Hôpital Tenon, 4, rue de la Chine, 75020 Paris. Tél. 40306618.

*** Direction des affaires médicales (directeur Jean de Savigny), Bureau des urgences et des gardes, 3, avenue Victoria, 75004 Paris. Tél. 45443939.

(1) En lle-de-France, il existe quatre SAMU départementaux:

75. Ville de Paris: Hôpital Necker, tél. 45675050 ;

92. Hauts-de-Seine: Hôpital Raymond-Poincaré, tél. 47417911 ;

93. Seine-Saint-Denis: Hôpital Avicienne, tél. 48311515 ;

94. Seine-et-Marne: Hôpital Henri-Mondor, tél. 48989242.

(2) SCPRI, 44, chemin de Ronde, BP 35, 78110 Le Vésinet - Tél. 39760432.

(3) Hôpital Percy, 101, avenue Henri-Barbusse, 92141 Clarmart - Tél. 46452104.

(4) Institut Gustave-Roussy, 39, rue Camille-Desmoulins, 94108 Villejuif - Tél. 45594909.

(5) Centre international de radiopathologie, Institut Curie, 26, rue d'Ulm, 75005 Paris Tél. 43291242 , poste 3631 . 


\section{A. POTENTIEL D'HOSPITALISATION EN 1987}

Nous avons enquêté auprès de 28 services susceptibles d'être impliqués et situés dans 14 hôpitaux de l'Assistance publique de Paris (tableau I), en différenciant deux types de services et de lits: ceux dits de "haute technicité" et ceux dits "spécialisés". On aura une idée de la densité de personnel correspondante en donnant le prix de facturation, tiré du prix de revient constaté, correspondant à $4584 \mathrm{~F}$ et $2094 \mathrm{~F}$ par jour respectivement.

\section{Les lits de "haute technicité"}

Au nombre de 154 situés dans 22 services, ils sont représentés par:

- les unités stériles d'hématologie réservés habituellement aux patients en aplasie médullaire et/ou bénéficiant d'une greffe de moelle osseuse;

- les unités stériles des services de brûlés;

- les chambres "radioprotégées" des services de curiethérapie et de médecine nucléaire.

Ils peuvent accueillir des blessés ayant reçu de fortes doses de rayonnements ionisants et nécessitant donc des soins spécialisés et une surveillance intensive.

\section{Les lits "spécialisés"}

On a réuni sous ce terme les moyens d'hospitalisation des services d'hématologie, d'oncologie médicale, de radiothérapie et de brûlés.

Ces lits spécialisés peuvent servir de dégagement des lits de "haute technicité" aussi bien en service normal qu'en cas d'urgence collective.

Ainsi les Hôpitaux de Paris disposent de 533 lits "spécialisés" s'ajoutant aux 154 précédemment décrits, soit au total l'équivalent d'un établissement spécialisé de 687 lits. S'ajoutent les lits de tous les autres services de médecine et de chirurgie d'un ensemble hospitalier de près de vingt mille lits placé sous la même autorité administrative.

\section{B. POSSIBILITÉS D'HOSPITALISATION CONSTATÉES}

L'enquête a été effectuée en interrogeant les responsables sur l'occupation constatée de leurs services le dimanche 26 avril 1987 (jour dit “J1"), le mercredi 29 avril (J4), le vendredi $1^{\text {er }}$ mai (J6) et le lundi 4 mai (J9) en distinguant :

- lits effectivement libres ces jours-là;

- lits que l'on aurait pu libérer compte tenu de l'état des malades présents en transférant par anticipation certains patients hospitalisés vers d'autres lits, soit de "haute technicité" vers les "spécialisés", soit des "spécialisés" vers les "normaux", mais tout en restant dans le même service et sous la responsabilité de la même équipe médicale. 
NOTES

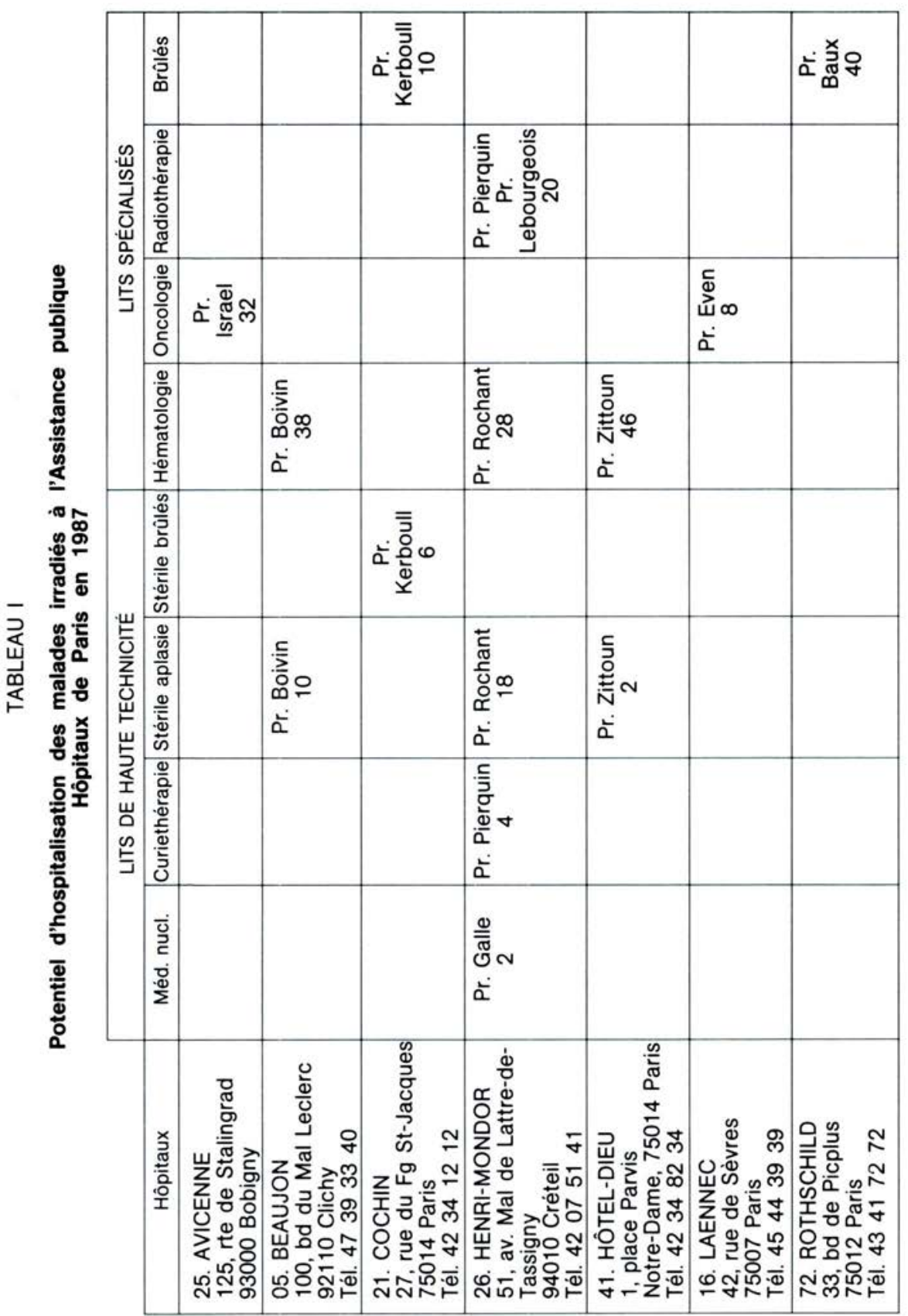


NOTES

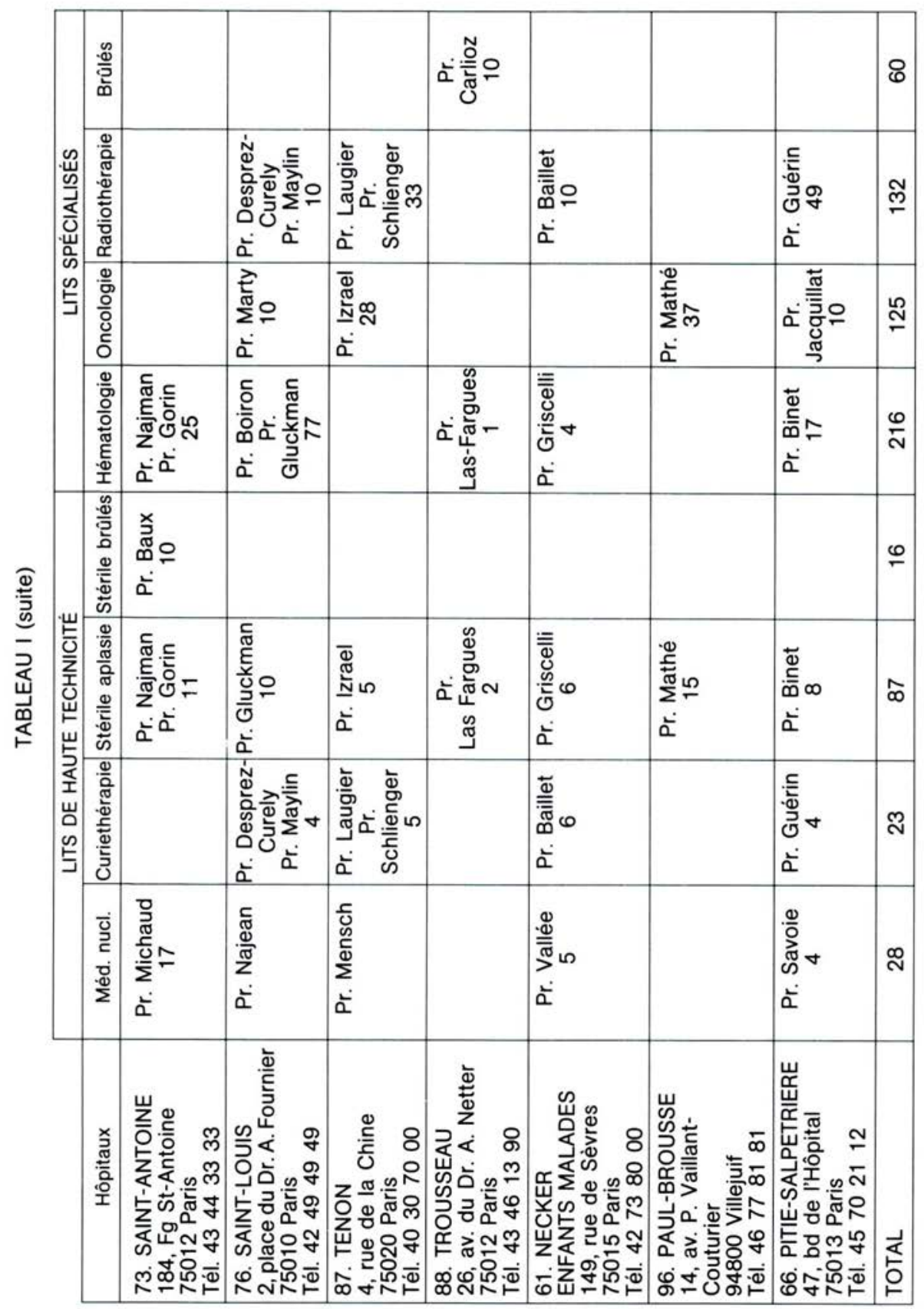


II existait le 26 avril 1987, jour anniversaire de l'accident, 25 lits immédiatement disponibles dans les unités stériles d'hématologie, de cancérologie ou de brûlés et 91 lits dans les services spécialisés.

Le 29 avril (J4), 33 lits de "haute technicité" et 58 lits spécialisés étaient disponibles. Compte tenu des variations d'activité liées aux fins de semaine et au $1^{\text {er }}$ mai, on constate que le nombre de lits disponibles variait pendant cette période de 71 à 156 (tableau II).

\section{TABLEAU ॥}

\section{Possibilités d'hospitalisation spécifique à l'Assistance publique Hôpitaux de Paris \\ constatées entre le 26 avril et le 4 mai 1987}

\begin{tabular}{|l|c|c|c|c|}
\hline \multicolumn{1}{|c|}{ Type de lits } & $\begin{array}{c}\text { Dimanche } \\
26 / 04\end{array}$ & $\begin{array}{c}\text { Mercredi } \\
29 / 04\end{array}$ & $\begin{array}{c}\text { Vendredi } \\
01 / 05\end{array}$ & $\begin{array}{c}\text { Lundi } \\
04 / 05\end{array}$ \\
\hline "Haute technicité" & $25+11$ & $13+20$ & $4+7$ & $10+11$ \\
\hline "Spécialisés" & $91+30$ & $40+18$ & $89+24$ & $46+41$ \\
\hline Total des lits disponibles & 156 & 71 & 117 & 97 \\
\hline
\end{tabular}

Le premier chiffre est le nombre de lits vides le jour considéré.

Le second est celui des lits susceptibles d'être dégagés au sein d'un même type de lits: le total des lits disponibles tient compte des transferts des malades d'un secteur à l'autre.

A Tchernobyl, 237 personnes ont été considérées comme atteintes de syndrome aigu d'irradiation et hospitalisées soit à Kiev, soit surtout à Moscou. Parmi elles, 42 avaient reçu plus de 4 Gy, 55 entre 4 et 2 Gy, les autres ayant reçu entre 2 et 0,8 Gy. Seuls, les irradiés à plus de 2 Gy relevaient d'une hospitalisation en milieu spécialisé.

Donc, les Hôpitaux de Paris sont susceptibles de faire face, à eux seuls et sans aucun problème, à un accident type "Tchernobyl".

En situation réelle d'accident, la mobilisation des moyens aurait permis d'accroître cette capacité d'hospitalisation en transférant des patients de services de "haute technicité" vers des services spécialisés et des services de médecine et de chirurgie et de faire face à un accident aux conséquences plus importantes. 